\title{
Transformations poétiques dans Les Mots secrets de Louise Dupré
}

\section{Annie Tanguay}

\section{(2) OpenEdition}

1 Journals

Édition électronique

URL : http://journals.openedition.org/genesis/4023

DOI : 10.4000/genesis.4023

ISSN : 2268-1590

Éditeur :

Presses universitaires de Paris Sorbonne (PUPS), Société internationale de génétique artistique littéraire et scientifique (SIGALES)

Édition imprimée

Date de publication : 1 juin 2019

Pagination : 55-68

ISSN : 1167-5101

\section{Référence électronique}

Annie Tanguay, «Transformations poétiques dans Les Mots secrets de Louise Dupré », Genesis [En ligne], 48 | 2019, mis en ligne le 01 juin 2020, consulté le 25 janvier 2021. URL : http:// journals.openedition.org/genesis/4023; DOI : https://doi.org/10.4000/genesis.4023 


\section{Transformations poétiques dans Les Mots secrets de Louise Dupré}

$\mathrm{A}^{\mathrm{u}}$ Québec, au début des années 2000, La courte échelle ${ }^{1}$ crée la collection «Poésie». En 2002 et 2003 , douze poètes québécois de renom composent des recueils de poèmes originaux destinés aux adolescents. Aucun autre recueil ne s'ajoutera par la suite.

L'esthétique des recueils de cette collection est assez conforme à ce que l'on peut trouver dans la poésie pour adultes. Les illustrations, souvent des eaux-fortes, occupent moins de place qu'elles ne le font généralement dans la littérature pour la jeunesse et entrent en dialogue avec les textes sans les réduire à une seule lecture. Mais les recueils de cette collection sont plus narratifs que beaucoup de recueils pour adultes. Un personnage les traverse, liant les textes les uns aux autres. Cela facilite la lecture sans pour autant diminuer la valeur poétique des livres.

C'est dans cette collection qu'à l'hiver 2002, l'écrivaine québécoise Louise Dupré 2 publiera son recueil de poésie pour adolescents Les Mots secrets (fig. 1). Elle est auteure d'une vingtaine de livres, dans différents genres littéraires, qui lui ont valu de nombreux prix et distinctions. Les Mots secrets est le seul qui s'adresse à la jeunesse.

Lorsque Sylvie Massicotte 3 , la directrice de la collection «Poésie», a demandé à Louise Dupré d'écrire des poèmes destinés aux adolescents, celle-ci a d'abord décliné l'invitation. Elle ne voyait pas ce qu'elle pouvait écrire «pour» les adolescents, mais elle a finalement choisi de relever le défi en écrivant «à partir» de la petite fille qu' elle était : «J'étais une enfant rêveuse. Les mots m'ont donné une certaine réalité, m'ont donné l'impression d'exister, m'ont sauvée, ont été mon lien à la vie et m'ont aidée à continuer », confiait-elle lors du lancement de la collection à l'hiver 2002. Bien qu'elle s'adresse à un nouveau public, son écriture lui semble être « aussi authentique ${ }^{4}$ » que dans ses autres livres.
Annie Tanguay

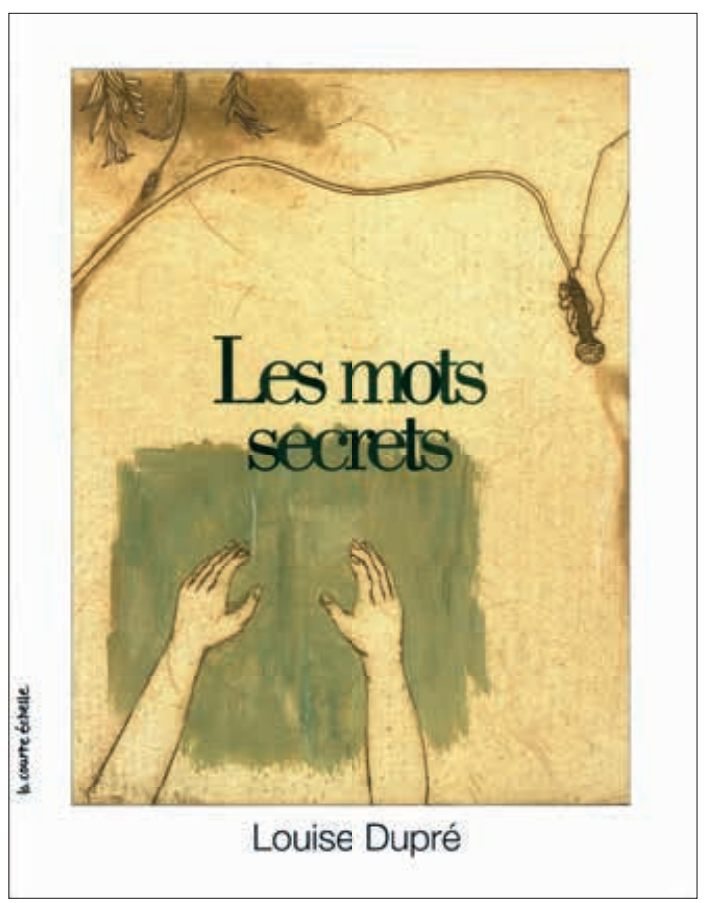

Fig. 1 : Couverture du recueil Les Mots secrets $(13,5 \times 18 \mathrm{~cm})$ de Louise Dupré, illustré d'un détail de l'eau-forte Microphone de Jean-Benoît Pouliot.

1. Créée en 1978, cette maison d'édition travaille à la promotion d'auteurs québécois pour la jeunesse.

2. Je voudrais remercier Louise Dupré pour sa confiance et sa disponibilité. C'est pour moi un honneur que de travailler à partir de ses archives littéraires, dans le cadre de mes recherches postdoctorales. Professeure associée à l'Université du Québec à Montréal (UQAM), Louise Dupré est également membre de l'Académie des lettres du Québec et de la Société royale du Canada. En 2014, elle a reçu l'Ordre du Canada «pour son apport à la littérature québécoise en tant que poète, romancière, dramaturge, essayiste et professeure».

3. Sylvie Massicotte a publié des nouvelles et un récit destiné à un public adulte, de même qu'une dizaine de romans pour la jeunesse à La courte échelle, où elle a été directrice littéraire de la collection «Poésie». Elle dirige de nombreux ateliers d'écriture et est chargée de cours au Département d'Études littéraires de l'UQAM.

4. Louise Dupré dans Gisèle Desroches, «Jeunesse de la poésie», Le Devoir, 9 février 2002, p. D1. 


\section{Le dossier génétique des Mots secrets}

De plus en plus d'écrivains lèguent leurs archives de leur vivant. À l'automne 2016, Louise Dupré a commencé à faire don des siennes au Centre Anne-Hébert de la Faculté des lettres et sciences humaines de l'Université de Sherbrooke5. Le dossier génétique des Mots secrets est constitué de neuf tapuscrits plus ou moins annotés par la poétesse, d'un tapuscrit annoté par Sylvie Massicotte et Louise Dupré, d'un jeu d'épreuves 6 et du scénario de l'adaptation scénique par le metteur en scène André Perrier ${ }^{7}$. Les documents ont été classés en six chemises par l'auteure (voir Annexe 1). Aucun feuillet n'est daté, à l'exception du scénario, et les poèmes n'ont pas de titre 8 .

Quatre états dactylographiés sont particulièrement intéressants d'un point de vue génétique :

- Le tapuscrit 1.1, quoique très fragmentaire - il ne comporte que trois poèmes -, est précieux de par sa différence de ton; il est écrit à la deuxième personne, alors que le tapuscrit suivant rend compte d'un changement majeur de l'énonciation, avec des poèmes écrits à la première personne. Bien que ce tapuscrit 1.1 ne soit pas daté, les transformations entre les différents états disponibles permettent d'établir qu'il s'agit là du document le plus ancien du dossier génétique. Par ailleurs, Louise Dupré a plus d'une fois confié en entrevue avoir d'abord composé son recueil à la deuxième personne avant de choisir la première. Quant aux manuscrits préalables, ils n'ont vraisemblablement pas été conservés.

- Le tapuscrit 1.2 contient vingt-deux des vingt-cinq poèmes du recueil. On y trouve somme toute peu d'annotations de la poétesse, mais elles témoignent notamment des thèmes qu'elle souhaite aborder. Par exemple, dans la marge inférieure du poème 21 , «Il m'arrive d'imaginer des paroles », elle écrit différents mots-clés qui orienteront son écriture, comme «amitié», «trahisons», «mots», «larmes», «abri », «savoir», «lumière».

- Le tapuscrit 1.3 est le premier état complet et comporte plusieurs annotations 9 .

- Le tapuscrit 3.1 contient des notes de l'éditrice et d'autres de Louise Dupré. Cette dernière tient compte de plusieurs remarques de Sylvie Massicotte pour ensuite retravailler ses poèmes.

Afin d'éclairer le processus créateur de l'auteure, j' analyse quatre des vingt-cinq poèmes du recueil, et renvoie à d'autres poèmes lorsque cela est pertinent. Mais tout d'abord, un mot sur la pratique d'écriture de Louise Dupré.

\section{La lecture comme mise en écriture (ou relance de l'écriture)}

Pour l'écriture de son recueil pour la jeunesse, Louise Dupré, comme à son habitude, écrit en lisant des textes d'auteurs créateurs : «J'écris toujours le matin en commençant par de la lecture parce que, curieusement, les mots me viennent avec les mots des autres », confiait-elle ${ }^{10}$. Quelques notes manuscrites consignées sur un feuillet quadrillé11 rendent compte d'une de ses lectures.

L'auteure note sur ce feuillet (fig. 2) près d'une vingtaine d'extraits du recueil de poésie Orbites de Martine Audet 12. Elle inscrit une première citation, au centre du feuillet, tirée des deux premiers vers du recueil : «<marteler $>$ les pluies d'une question impossible13», sous laquelle elle note six autres fragments. Elle en écrit d'autres encore au haut du feuillet. Différents fragments, dont «piquent la

5. Le fonds d'archives Louise Dupré (P78) est conservé au Service des bibliothèques et archives de l'Université de Sherbrooke (SBAUS).

6. Seul le texte de Louise Dupré apparaît dans ces épreuves; les eauxfortes de l'artiste graveur Jean-Benoît Pouliot n'y figurent pas encore.

7. Pour ce spectacle, le metteur en scène, comédien et dramaturge francocanadien a sollicité la collaboration d'élèves de $4 \mathrm{e}$ année du primaire afin d'adapter le texte, en ajoutant quelques dialogues et jeux de mots à travers les poèmes. Produit par le théâtre Triangle Vital, le spectacle, destiné à des enfants de 8 ans et plus, a été présenté à l'automne 2017 dans diverses maisons de la culture de Montréal.

8. Les numéros des poèmes utilisés dans cet article correspondent à l'ordre de présentation des textes dans le recueil (voir «Liste des incipit des poèmes publiés », Annexe 2). Quant aux numéros des feuillets entre parenthèses, ils permettent de situer les poèmes dans les tapuscrits.

9. Pour les états 1.1 à 1.3 , l'ordre des textes diffère de la version finale. Dans le tapuscrit 1.3 , seuls cinq des vingt-cinq poèmes ont conservé le même ordre de présentation, dont les deux premiers textes. Dès l'état 2.1, les textes sont classés en fonction de l'ordre définitif.

10. Sylvain Marotte (réalisateur), Au fil des mots. Louise Dupré, poète, Productions Téridan Inc., 2005, 25 min.

11. Ce feuillet est conservé dans la chemise 3 avec deux autres feuillets de notes non numérotés, ainsi que les tapuscrits 3.1 et 3.2 .

12. Orbites, Montréal, Le Noroît, 2000.

13. «chaque nuit les étoiles martèlent du vide / comme les pluies d'une question impossible» (Martine Audet, Orbites, op. cit., p. 9). 


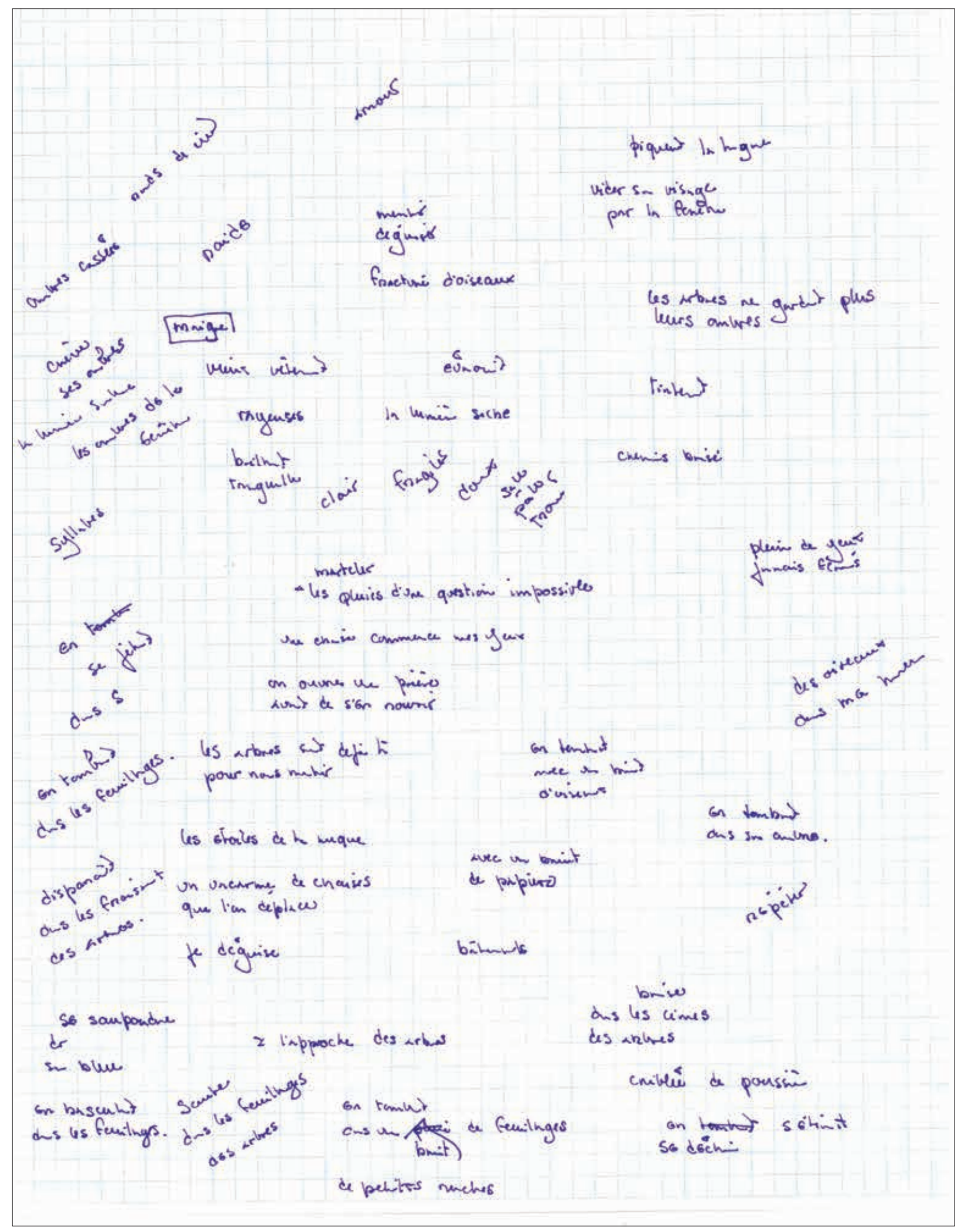

Fig. 2 : Feuillet de notes manuscrites (chemise 3). Fonds Louise Dupré, SBAUS. 
langue», «syllabes» et «des oiseaux dans ma bouche ${ }^{14}$ », sont liés au langage, à la parole, thèmes centraux dans Les Mots secrets. Les extraits sont le plus souvent tronqués; Louise Dupré note rarement les vers en entier, n'écrivant que les mots qui l'interpellent alors. À plusieurs endroits à gauche et au bas du feuillet, elle écrit quelques mots sur les arbres, sur les feuillages des arbres, dont «en tombant dans les feuillages», «en basculant dans les feuillages», «à l'approche des arbres», "saute dans les feuillages des arbres», «brise dans la cime des arbres», que l'on ne trouve pas textuellement dans le recueil de Martine Audet ${ }^{15}$. Une idée se développe alors; la mise en écriture suit son cours.

Ces annotations servent à la poétesse dans l'une des réécritures du deuxième texte de l'ensemble. Dans le tapuscrit 3.1, annoté par Sylvie Massicotte et Louise Dupré, l'éditrice suggère à l'auteure de terminer son poème avec le vers «là, juste là où le ciel se déchire», expliquant que la finale lui semblerait «plus forte». Louise Dupré envisage alors de modifier les trois derniers vers : «jusqu'à laisser apercevoir / la lumière rosée / au creux des nuages » (3.1, f. 2). Elle inscrit différentes formules, dont «en tombant se déchire », « voir / une lumière rosée / qui ne ment pas», «en tombant / dans la lumière $<$ feuillages $>$ / des ar / d'un érable / de ma», «dans les feuillages < la lumière $>/$ des arbres», «en tombant / se brise» et «menace de se déchirer» (3.1, f. 2 ; fig. 3). De ces essais, elle ne conservera que l'idée de la chute : «là, juste là où le ciel / en tombant / risque de se déchirer» (4.1, f. 2$)$.

Lire donne à Louise Dupré une impulsion dans sa propre écriture. Cependant, si elle consigne des extraits de différentes œuvres, elle les reprend rarement dans ses textes. Sans l'enquête génétique, il aurait été impossible de dire que, pendant qu'elle compose ses poèmes, elle lit notamment le livre de Martine Audet. Comme pour ses autres livres, Dupré écrit en compagnie d'autres écrivains; elle écrit «dans les trous de ce qu'ils disent 16 ».

\section{«Au cour du silence» : l'altérité}

Louise Dupré avait d'abord choisi d'écrire ses poèmes à la deuxième personne. Plus qu'un dialogue avec l'autre, l'adresse se rapproche d'un dialogue du sujet-narrateur (ou personnage) avec lui-même. Le «tu» s'apparente ici davantage à un double de la poétesse ${ }^{17}$.
Aucun autre tapuscrit que le 1.1 ne présente un tel point de vue. Sur les trois feuillets de cet état, la dynamique est alors complètement différente, et ce, dès les premiers vers du poème d'ouverture, «Au cœur du silence», où Louise Dupré emploie le verbe «perdre», plutôt que «percer» : «Au cœur du silence / il y a les mots / tous les mots de lait / que tu as appris / à l'âge tendre où tu perdais / tes dents » (1.1, f. 1 , fig. 4 ; je souligne).

Le premier rapport aux mots évoqués ne remonte pas à la petite enfance comme ce sera le cas dans les autres états 18 , mais correspond ici à l'entrée de l'enfant à l'école primaire, à ce moment où il apprend à lire et à écrire. D'ailleurs, la fin du poème d'ouverture présente le recueil comme un guide : «Les mots sont à toi / pour ta première danse / ou pour ce poème / que tu liras peut-être / en secret / avec l'impression de rejoindre / l'écho de ta propre voix ${ }^{19}$ » $(1.1$, f. 1 ; je souligne). Le ton est alors plus éducatif, voire maternel. L'adresse à la deuxième personne, même quand elle permet à l'énonciateur de s'adresser à lui-même, introduit une distance, qui tend à être interprétée par l'enfant comme un surplomb.

La directrice de la collection faisait ainsi remarquer à Louise Dupré que cette adresse pouvait apparaitre comme une voix de l'autorité. Selon Sylvie Massicotte, plus le protagoniste est jeune - Louise Dupré écrit pour des jeunes de 11 à 14 ans - plus le «tu» peut sembler moralisateur : «La voix semble venir de l'extérieur plutôt que de l'intérieur comme

14. «les clochers du soleil piquent la langue», «syllabes ardentes » et «des oiseaux s'affolent dans ma bouche» (ibid., p. 23, 55, 73).

15. On pourrait voir aux nombreuses notes sur les feuillages un lien avec deux vers d'Orbites - «bien des paroles se cachaient / dans les feuillages » (ibid., p. 28) -, que Louise Dupré n'a pas consignés.

16. Louise Dupré dans Stéphane Lépine, Un chœur qui bat. Propos et entretiens sur l'œuvre de Louise Dupré et la production de la pièce Tout comme elle mise en scène par Brigitte Haentjens, Montréal, Publications Sibyllines, 2006, p. 11. Le document est maintenant disponible en ligne : $<$ http://sibyllines.com/uploads/tce.pdf >.

17. C'est ainsi qu'elle explique sa pratique d'écriture à l'animateur Stanley Péan du magazine littéraire Bouquinville, diffusé sur les ondes de Radio-Canada, le 23 mai 2004. Elle venait alors de faire paraître son recueil de poèmes Une écharde sous ton ongle.

18. «Au cœur du silence / il y a les mots / tous les mots de lait / lentement appris / à l'âge où je perçais mes dents » (ibid., p. 9; je souligne).

19. «Les mots sont à moi / pour ma première danse / ou pour des poèmes / que je lis / avec l'impression de toucher / l'écho d'une voix / toute neuve en moi» (ibid., p. 9). 
Je connais des mots larges

comme les vagues

que le vent souffle sur la mer

les matins de pluie

d'autres ressemblent à des maisons

chaudes à l'abri du vacarme

Il m'arrive souvent de chercher

refugeen moi (Nus faible)

en esperant-un peu de paix

car je ne sais plus

pour qui bat mon coeur

sous mon chandail

Et ce visage trop grand pour moi

je le regarde

avec une moue boudeuse dans le miroir

avant de tourner les yeux

vers la fenêtre cnibli d'o $f_{n}$ higum

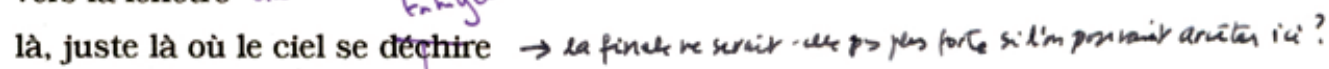

\section{jusqu'àtaisser apercevoir sorokl'}

la lumière rosée

au creux des nuages
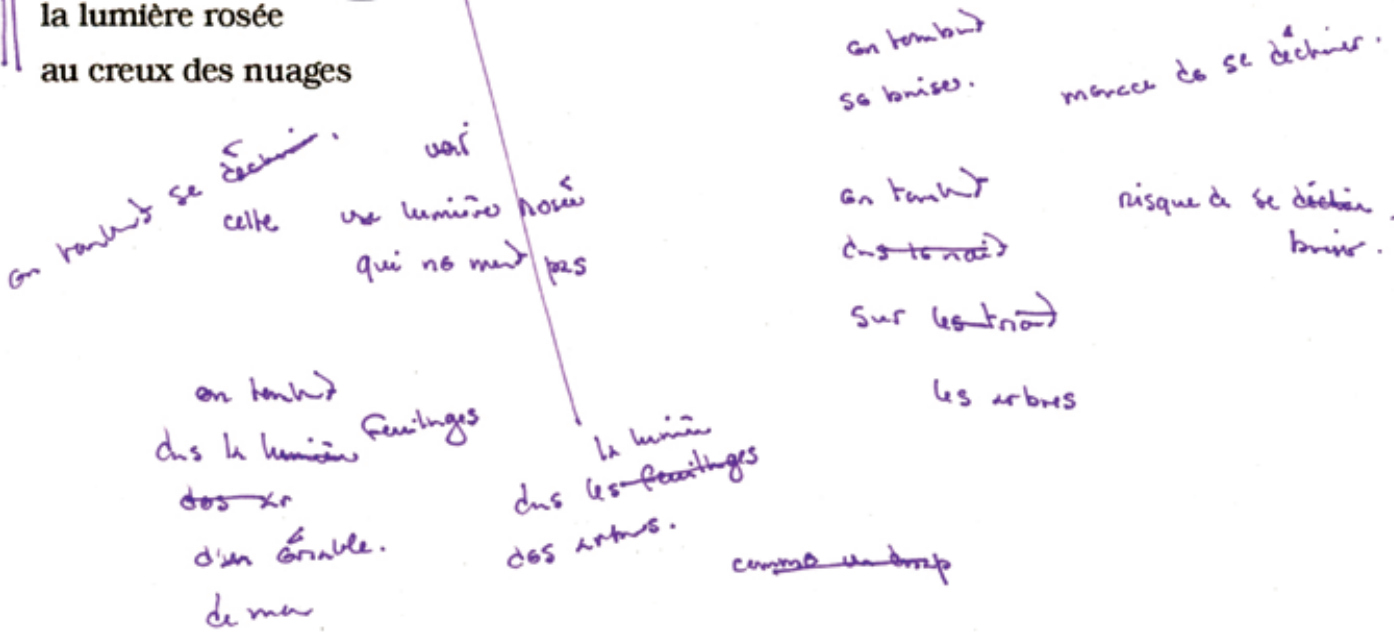

Gn tant

Cing tonat

risque d se dictir.

Sur lestrom

les arbors

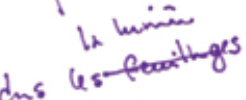

dos artors.

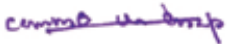

Fig. 3 : Tapuscrit de «Je connais des mots aussi larges» : à l'encre noire, les commentaires de l'éditrice, et à l'encre mauve, les modifications de Louise Dupré $(3.1$, f. 2). Fonds Louise Dupré, SBAUS. 
Au coeur du silence

il y a les mots

tous les mots de lait

que tu as appris

à l'âge tendre où tu perdais

tes dents

Tu les roulais sur ta langue

avant de les cracher

comme de gros ballons

capables de voler jusqu'aux étoiles

Car tu aimes les mots

et même si tu as grandi

ils se sont incrustés

dans le sucré de ta chair

tu les conserves en toi

là où personne ne pourra

te les arracher

Les mots sont à toi

pour ta première danse

ou pour ce poème

que tu liras peut-être

en secret

avec l'impression de rejoindre

l'écho de ta propre voix

Fig. 4 : Tapuscrit de «Au cœur du silence» $(1.1$, f. 1). Fonds Louise Dupré, SBAUS. 
l'auteur le souhaiterait. J'en suis venue à la conclusion que l'enfant n'avait pas cette capacité d'auto-analyse dont il fera preuve à l'adolescence 20 . $\gg$ Elle a tenté de valider son impression auprès d'une amie psychologue qui lui expliquait que l'auto-analyse chez l'enfant - l'intégration des valeurs, des diktats, des règles de conduite - n'existe pas encore.

Louise Dupré réécrit ses poèmes à la première personne, mais elle conserve dans trois poèmes (les poèmes 8,22 et 23) une adresse à la deuxième personne, qui s'apparente cette fois à un dialogue avec l'autre. Ces adresses disparaîtront cependant au fil des réécritures. Dans le poème 23, «Je ne crois plus aux fées », la deuxième personne rend compte de la relation d'amitié vécue par le sujet-narrateur. Ce dernier souhaite trouver le moyen de ne pas oublier certains bons moments : «tes plus beaux sourires / la carte postale de tes dernières vacances / et les confidences que tu murmures à mon oreille» $(1.3$, f. 23$)$. Louise Dupré conclut alors son poème sur ces trois vers : «C'est toi qui m'auras appris < apprends > / la langue vivante / de l'amitié» $(1.3$, f. 23). Dans le tapuscrit 3.1, à droite de ce passage, l'éditrice pose une question à l'auteure : «Trop explicatif? », et lui signale par ailleurs que «certains lecteurs 21 disent préférer quand ça ne prend pas le "tu" » (3.1, f. 23). Les corrections de Louise Dupré vont dans le sens des remarques de l'éditrice : «sa photo / la carte postale de ses dernières vacances / et les confidences / chuchotées lentement à mon oreille» $(4.1$, f. 23$)$. La poétesse choisit en outre de retirer les trois derniers vers. Ces modifications centrent davantage le propos sur l'intériorité du personnage.

\section{«Le matin se lève toujours trop tôt » : papillon en éveil}

Le huitième poème, «Le matin se lève toujours trop tôt», présente plusieurs modifications et annotations intéressantes. Dans tous les états qui précèdent les épreuves (de 1.2 à 4.2), le sujet-narrateur s'adresse à un «tu». Mais, contrairement au poème 23 qui célèbre l'amitié, le huitième poème s'apparente à une déclaration d'amour :

le verbe aimer/conjugué au futur / le contour d'un visage / dont j'ignore les traits / Un jour, je sais 22 / tu viendras dans ma vie / je te reconnaîtrai à ton sourire / suspendu à la mer $(1.2$, f. 8$)$.
Louise Dupré modifie légèrement ces quelques vers, mais sans jamais retirer l'adresse à la deuxième personne ${ }^{23}$. L'adresse ne disparaît qu'aux épreuves; il semble ainsi manquer l'état dactylographié expédié à l'éditeur, à moins qu'il manque au dossier d'autres jeux d'épreuves. Dans le reste du recueil, c'est l'image d' «un ami» parti «pour cette ville si loin ${ }^{24} \gg$ qui prime. La question amoureuse, comme d'autres facettes de la vie adolescente, est évoquée, mais sans que cela soit dirigé vers un destinataire précis. En éliminant l'adresse à la deuxième personne, le sujet-narrateur se projette simplement dans l'avenir et rêve d'une passion prochaine.

Dans le tapuscrit 1.2, Louise Dupré ajoute, à droite de deux passages, la mention «adulte»: «pour les langues qui savent apprivoiser (protéger) blesser / leurs voyelles» et «il faudra me fier à moi / puisque les mots de l'amour / ne s'apprennent pas / même dans les beaux livres » (1.2, f. 8; fig. 5). Aucune modification n'est alors apportée. Dans le tapuscrit suivant, elle cherche à ajuster sa voix à celle

20. Sylvie Massicotte, «2017 : Réflexion». Il s'agit là d'un feuillet qu'elle m'a remis le 8 mars 2017. D'autres recueils de la collection «Poésie» ont une adresse à la deuxième personne, mais ils s'adressent à un lectorat un peu plus âgé que celui des Mots secrets.

21. Le recueil a vraisemblablement été soumis à un comité de lecture et l'éditrice fait part à l'auteure des remarques des lecteurs.

22. Dans les autres états, l'auteure élimine le «je sais», atténuant de ce fait les marques de la modalité du savoir catégorique. Louise Dupré en supprime d'ailleurs un autre dans le poème 5 , «Le chagrin ne ménage personne» : «Le chagrin ne m'épargne pas / le chagrin, je sais / n'épargne personne» $(1.3$, f. 11$)$.

23. « le verbe aimer / conjugué au futur / le contour d'un $<$ e $>$ visage $<$ silhouette encore dans l'ombre te brouillard inconnue $>$ / dont j'ignore les traits / Un jour, tu viendras dans ma vie / je te reconnaîtrai à ton sourire / suspendu à la mer» $(1.3$, f. 8$)$; «le verbe aimer / conjugué au futur / le contour d'une silhouette / encore inconnue / Un jour, tu viendras / dans ma vie / je te reconnaîtrai à ton sourire / suspendu à la mer » (2.1-2.2, f. 8); «le verbe aimer / conjugué au futur / le contour d'une silhouette/encore inconnue / Un jour, tu viendras / dans ma vie / je te reconnaîtrai à ton sourire < écharpe lèvres > / suspendu < e > à la mer » ( 3.1 , f. 8$)$; «le verbe aimer / conjugué au futur / le contour d'une silhouette / encore inconnue / Un jour, tu viendras / dans ma vie / je te reconnaîtrai à tes cils / suspendus à la mer» $(4.1$, f. 8$)$; «le verbe aimer / conjugué au futur / le contour d'une silhouette/encore inconnue/Un jour, tu viendras / dans ma vie / je te reconnaîtrai à tes lèvres / suspendues à la mer» $(4.2$, f. 8$)$; «le verbe aimer / conjugué au futur / le contour d'une silhouette / encore inconnue / mais qui viendra un jour / dans ma vie / je la reconnaîtrai à ses lèvres / suspendues à la mer» (5, f. 8; Louise Dupré, op. cit., p. 18).

24. Ibid., p. 11 . 
Le matin se lève toujours trop tôt

car le coeur ne vibre

que la nuit, dans le noir

qui abrille les rêves

un beau velours tendu

à la fenêtre, le verbe aimer

conjugué au futur

le contour d'un visage

dont j'ignore les traits

Un jour, je sais

tu viendras dans ma vie

je te reconnaitrai à ton sourire

suspendu à la mer

ou à ta passion

pour les langues qui savent apprivoiser (protéger) blesser || kculte

leurs voyelles

il faudra me fier à ces antennes

de papillon qu'on sent

parfois sous la peau

il faudra me fier à moi

puisque les mots de lamour

ne s'apprennent pas

même dans les beaux livres (25)

nciulte.

ok

Fig. 5 : Tapuscrit de « Le matin se lève toujours trop tôt » $(1.2$, f. 8$)$. Fonds Louise Dupré, SBAUS. 
de son public. Elle élimine d'abord le verbe «savoir» du premier passage : «pour les langues qui ont apprivoisé $<$ font chanter $>/$ leurs voyelles 25 » $(1.3$, f. 8$)$. Cette réécriture atténue l'idée d'une langue savante et réflexive et fait ressortir l'aspect musical des voyelles que tout un chacun peut reconnaître. Dans le second passage, le sujet-narrateur n'a probablement pas eu la chance de parcourir tant de «beaux livres » et n'aurait peut-être pas non plus eu la sagesse de dire que ceux-ci ne pouvaient lui apprendre «les mots de l'amour».

Louise Dupré essaie ensuite une formule où le sujet se fait inventeur : «i $<\mathrm{I}>1$ faudra me fier à moi / puisqu'il faut inventer/ses propres mots < paroles serments > d'amour» (1.3, f. 8). Elle maintient cette image dans les états 2.1 et 2.2, mais elle choisira de terminer son poème sur la métaphore du papillon qu'elle ajoute au bas du feuillet : «Il me faudra me fier à < ces $<$ frêles minces $>$ antennes < étroites fragiles légères dressées aux aguets $>/<$ quand $>$ contrer la solitude $<$ l'habitude du silence $>$ / à calmer / de papillon nocturne $<$ en éveil $>26$ » $(3.1$, f. 8 ; fig. 6$)$. Le papillon, ainsi que le lecteur qui entre dans l'adolescence, vit de grands changements et s' «éveille» au monde sous une nouvelle forme.

\section{«J'ai l'habitude d'écrire» : une part de mystère}

À deux endroits dans le recueil, Louise Dupré éliminera le mot «école 27 ». Dans le premier état du poème 22, «J'ai l'habitude d'écrire », le sujet-narrateur «trace les lettres » du prénom de l'être aimé «sur un saule / rêveur dans le parc / près de l'école / ou sur [s]a main gauche» $(1.2$, f. 12). Dans les états subséquents, la poétesse délaisse le «saule rêveur» et le remplace par un «cahier d'exercices» : «J'ai l'habitude d'écrire / ton prénom / sur ma main gauche / ou dans mon cahier d'exercices / à l'école» (3.1, f. 22). Sylvie Massicotte lui suggère alors de supprimer le dernier vers de l'extrait, ce à quoi Louise Dupré consent puisqu'il ne figure pas sur le tapuscrit suivant. Malgré tout, avec «cahier d'exercices», l'univers scolaire ne disparaît pas complètement, mais en éliminant le mot «école» et l'allusion au parc (lieu fréquenté majoritairement par des enfants), le poème est moins figé, et ce, tant au niveau de la temporalité que des lieux.

Ce poème est le seul autre texte à employer un «tu», qui traduit, comme dans le poème 8 , un rapport amoureux : «comme s'il suffisait d'écrire / ton prénom / pour que tu m'aimes/avec mon corps désaccordé/et ces petits boutons / qui narguent mes joues » (1.2, f. 12). Malgré quelques corrections mineures, ces vers resteront sensiblement les mêmes jusqu'à la lecture de l'éditrice. Louise Dupré éliminera ensuite l'adresse tout en s'attachant davantage aux sentiments du sujet-narrateur : «comme s'il suffisait de tracer les lettres / d'un nom chéri / pour qu'on m'aime / avec mon corps désaccordé / et ces boutons qui ne cessent / de me narguer» $(4.2$, f. 12). Les boutons ne narguent plus uniquement les joues du sujet-narrateur, mais tout son être. Il devra apprendre à composer avec son nouveau physique et tenter d' «apprendre à sourire / malgré [lui]» (4.2, f. 12).

Au fur et à mesure des réécritures, Louise Dupré ajoute une part de mystère à son poème en en retravaillant la seconde moitié. Selon le poète et pédagogue français JeanPierre Siméon, même si un poème ou un recueil s'adresse à des enfants, il ne faut «pas céder sur ce qui fait l'intérêt de la poésie : susciter l'étonnement, l'interrogation, la compréhension différée, imprévue... une compréhension qui doit réinventer sans cesse ses moyens $28 »$. François David, le fondateur des Éditions Møtus, maison d'édition française qui se consacre principalement à la littérature jeunesse, va dans le même sens que Siméon en abordant l'idée du respect du public. Il faut traiter l'enfant ou l'adolescent «comme un vrai lecteur ${ }^{29}$ », en gardant dans le poème une richesse lexicale sans pour autant proposer un sujet trop difficile pour lequel il n'aurait pas le bagage culturel suffisant.

Sur le premier tapuscrit, Louise Dupré aborde déjà le thème du dédoublement, mais de manière simplifiée, voire enfantine, à travers l'idée de la «fête» et d'une «formule

25. «pour les langues qui font < laissent > chanter / leurs voyelles» $(2.1$, f. 8$)$; " pour les langues qui laissent chanter / leurs voyelles » $(2.2,3.1-4.2$, f. 8$)$; «pour les langues laissant chanter / leurs voyelles » (5, f. 8 ; ibid., p. 18).

26. « Il faudra me fier à ces antennes / qu'on sent parfois sous la peau / ces frêles antennes / de papillon en éveil» (4.1-5, f. 8 ; ibid., p. 18).

27. Dans le troisième poème, «Je joue parfois avec les lettres », elle ne retiendra pas le second vers de cet extrait : «Je me demande s'il pense à moi / en revenant de l'école» $(1.3$, f. 4$)$.

28. Manuela Barcilon et Annick Lorant-Jolly, «Entretien avec Jean-Pierre Siméon », La Revue des livres pour enfants, $\mathrm{n}^{\circ} 258,2011, \mathrm{p} .78$.

29. Manuela Barcilon et Annick Lorant-Jolly, «Une maison d'édition dédiée à la poésie pour la jeunesse : Møtus. Entretien avec François David», La Revue des livres pour enfants, ${ }^{\circ}$ 258, 2011, p. 94. 
Le matin se lève toujours trop tôt

car le coeur ne vibre

que lanuit, dans le noir

recouvrant les rêves

ont un bear velours tendu

à la fenêtre, le verbe aimer

conjugué au futur

le contour d'une silhouette

encore inconnue

Un jour, tu viendras

dans ma vie
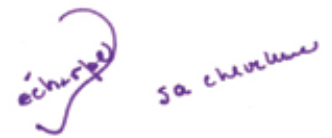

je te reconnaîtrai à ton sourire $\mathrm{km}$ pacile...
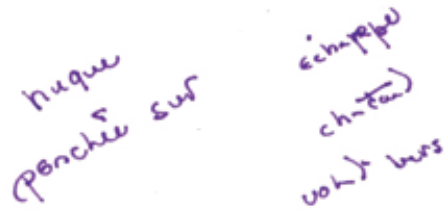
suspendừ à la mer

ou à ta passion

pour les langues qui laissent chanter

leurs voyelles

il faudra me fier àes antennes

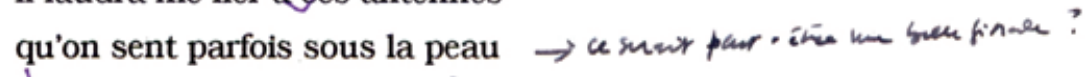

IIfaudra me fier à moi

puisqu'on doit inventer

ses propres serments d'amour
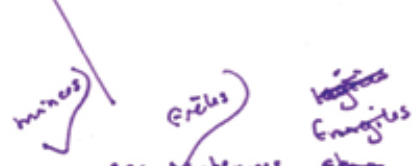

cos Rniennes

entor

drecusts

Lene a garts

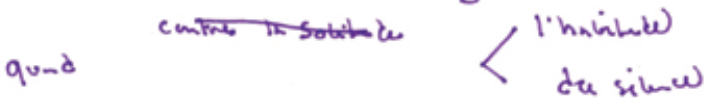

$x$ ealine

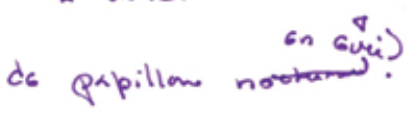

Fig. 6 : Tapuscrit de «Le matin se lève toujours trop tôt» : à l'encre noire, les commentaires de l'éditrice, et à l'encre mauve, les modifications de Louise Dupré $(3.1$, f. 8). Fonds Louise Dupré, SBAUS. 
magique» (1.2, f. 12). Sur l'état suivant (1.3, f. 12), elle précise que c'est par le regard, plutôt que par les traits ou le visage, qu'il est possible de traduire la beauté :

Chaque lettre est une fête $<$ philtre $>$ / chaque lettre est une formule/magique où j'a $<\mathrm{e}>$ perçois les traits $<$ un autre regard dense précis-> / d'un autre visage / pour l'instant caché / derrière mon visage $<$ derrière mon regard $>/$ et qui un jour $<$, je $>$ saura $<$ i $>$ / trouver les mots $<$ phrases $>$ pour dire / la beauté $<$ l'angle qu'il faut / pour voir dire traduire la beauté $>$

Le mot «talisman» (2.1 et suivants), qui implique une certaine forme de magie et qui est souvent associé à la protection du mauvais œil, vient ensuite remplacer «philtre». La poète essaie différents vers, mais ce n'est que sur l'état 4.2 que le terme «œil» est employé et que le concept de beauté est remplacé par celui d'acceptation : «Chaque lettre est un talisman / chaque lettre est un œil / clignant derrière mon regard / pour m'apprendre à sourire / malgré moi 30 » $(4.2$, f. 22$)$. D'ailleurs, sur l'un des deux feuillets manuscrits non numérotés sur papier quadrillé conservés dans la chemise 3 , des notes témoignent des hésitations de l'auteure qui cherche la bonne formulation :

et qui me voit

chaque lettre est un œil / eaché < qui se cache > / derrière

mon œil / gauche / pour regarder

qui cligne / derrière mon œil < dans mon œil > / pour

Les lettres du prénom de l'être aimé acquièrent ici un pouvoir insoupçonné, tout comme les mots dans un poème où les associations inattendues sont fréquentes, ce qui plaît généralement aux enfants.

\section{«Je ne trouve pas toujours» : le mot de la fin}

Dans le tapuscrit 1.2, le poème-clôture n'est vraisemblablement pas encore composé. Le recueil ne comporte que vingt-deux textes et les quatre derniers vers du poème final, «Il suffit d'ouvrir ma fenêtre » - le onzième dans le recueil -, sont assez différents de ce que l'on retrouvera par la suite : «e'est le jour / qui jettent une joie / nouvelle sur la erue $<$ mon petit monde $>/$ du jour $\gg(1.2$, f. 22$)$. Bien que ce dernier vers ne soit pas supprimé, Louise Dupré continue son poème comme s'il n'y était plus : «< que je recompose / lentement / à la manière / de ceux qui ne sont pas
< ont encore > / encore grands / du temps / pour grandir > (1.2, f. 22). Plutôt que le verbe «grandir», l'auteure optera, dans les autres états, pour celui d' «aimer $31 »$. Cela atténue l'idée du passage de l'enfance à l'adolescence, pour un sujetnarrateur qui souffre des changements qui se produisent en lui. Dans le tapuscrit 1.3, la poète songe cette fois à terminer son recueil avec l'avant-dernier poème, «Mes plus beaux rêves ». Les derniers mots du recueil auraient repris le titre de l'ensemble : «Et $d<D>$ ans ma tête/mon image finit par ressembler / à mes mots les plus secrets 32 » $(1.3$, f. 25$)$. Mais, dans ce texte, le sujet-narrateur est alors davantage replié sur lui-même, restreint au monde des rêves, aussi «beaux 33 » et positifs soient-ils.

Le poème-clôture, «Je ne trouve pas toujours », exprime bien que, pour mieux appréhender le monde qui l'entoure, le sujet-narrateur se tourne vers la poésie, vers cette forme d'écriture qui lui résiste et le charme à la fois : «Alors je lis des poèmes / avec des images / offertes comme les eaux / qui courent jusqu'à la mer / car les poèmes ont une peau / plus vibrante que ma peau 34 ». Le recueil se clôt alors sur une formule plus ouverte : «Les poèmes sont ma fenêtre / et mon autre visage 35 ». Par la poésie, le sujet peut être tout ce qu'il souhaite. Les mots permettent une forme de dédoublement, de multiplication des êtres, des choses. D'ailleurs, cet «autre visage» fait référence à cet autre - l'adolescent par rapport à l'enfant - qui habite désormais le sujet-narrateur, qui le fait se sentir étranger à lui-même. Les derniers vers ont tout de même subi quelques changements significatifs : «les poèmes sont mon autre visage < caché > (ma présence) / et ma ligne d'horizon» $(1.3$, f. 25$)$, «les poèmes sont mon autre visage/et ma ligne d'horizon » $(1.3$, f. $24 ; 2.2,2.3,3.1$, f. 25$)$ et «Les poèmes sont ma fenêtre $<$ une promesse $><$ mes pôles $>/$ et mon

30. «Chaque lettre est un talisman / chaque lettre est un œil invisible / clignant / derrière mon œil / pour me faire sourire / malgré moi » (Louise Dupré, op. cit., p. 35).

31. «La lumière jette une joie / sur mon petit monde / et chaque jour, je le recompose / à la manière de ceux / qui prennent du temps / pour aimer » (ibid., p. 21).

32. «Et mon image dans ma tête / finit par ressembler / à mes mots secrets » (ibid., p. 38).

33. Ibid., p. 38.

34. Ibid., p. 39

35. Ibid., p. 39. 
autre visage» (4.1, f. 25$)$. La «ligne d'horizon» a une limite liée à la vision. En éliminant ce dernier vers, le poème reste ouvert à tous les possibles.

Aucune ponctuation ne vient fermer le dernier poème, ni aucun autre texte du recueil. Le point est limitatif; sans point «le texte pouvait continuer, il s'enfonce dans le silence», m'a confié Louise Dupré36.

\section{Conclusion}

Par son recueil Les Mots secrets, Louise Dupré voulait aborder le malaise propre aux adolescents qui souvent ne se trouvent pas très beaux et ont l'impression que tout leur échappe :

J'avais envie d'aller chercher l'essentiel, ce qui transcende, ce qui reste une fois toutes les modes passées : l'envie d'être seul, le désir d'avoir des secrets, la constatation des changements du corps, l'expérience de la perte d'un ami, le goût de grandir, la révolte devant le monde actuel, etc. 37

La poétesse fait état dans ses textes des changements physiques et physiologiques vécus par les jeunes : l'acné, les jambes qui s'allongent, les tonalités de la voix qui changent, etc. Pour réussir à passer outre ces désagréments, le sujet « cherche un visage / ajusté au timbre de [s]a voix / un de ces visages / lents du matin / mais qu['il] pourrai[t] habiter / jusqu' au dernier soir 38 ». Dans le tapuscrit 1.3, à droite de ce fragment de «Personne ne me comprend», Louise Dupré inscrit à deux reprises le mot «sourire», deux fois le mot «rire», de même que les mots «bonheur» et «joie» $(1.3$, f. 7$)$. Ces termes positifs en marge viennent appuyer ce qu'elle écrit tout au long du recueil : il y a de l'espoir. Ce dernier mot vient d'ailleurs clôturer le cinquième poème, «Le chagrin ne ménage personne »: «ces mots humains / que j'aligne sur une feuille / pour créer un lexique / de l'espoir ${ }^{39}$ ».

Louise Dupré occupe cet espace de liberté qu'est la poésie. «On ne peut pas non plus ignorer que l'être humain a besoin d'une parole qui sonne vrai à son oreille 40 », ditelle. Par Les Mots secrets, elle travaille à ce que sa parole sonne juste pour son public. Cette rapide incursion «dans le laboratoire secret» de Louise Dupré, «dans l'espace intime d'une écriture qui se cherche 41 , témoigne de quelques opérations effectuées par la poétesse afin d'ajuster sa voix, d'écrire à partir de celle qu'elle était, tout en portant son regard d'adulte et d'auteure sur son passé. En écrivant ses poèmes au «je», elle favorise un sentiment de complicité entre le sujet-narrateur et le lecteur, afin que l'allocution ne renvoie pas à une voix adulte, à une figure d'autorité. L'énonciation à la première personne favorise ainsi l'identification empathique du jeune lecteur avec le sujet lyrique. Louise Dupré recentre à plusieurs endroits son propos sur l'intériorité du personnage, évitant les généralités et les clichés adolescents. Elle est par ailleurs sensible aux commentaires de son éditrice qui connaît bien le public à qui s'adresseront les poèmes. Et bien que son recueil s'adresse à des jeunes de 11 à 14 ans, son écriture n'est pas enfantine. Certaines images ont de quoi surprendre les lecteurs et le lexique employé est riche et varié. Comme pour ses autres livres, la poétesse écrit en lisant d'autres écrivains ; les mots des autres lui insufflent petit à petit le désir d'écrire. C'est un «dialogue muet 42 » qui s'établit alors, à l'image de celui qu'elle souhaite elle-même instaurer avec ses lecteurs.

36. Entretien accordé le 14 septembre 2016, à Montréal.

37. Louise Dupré dans Gisèle Desroches, «Jeunesse de la poésie », Le Devoir, 9 février 2002, p. D1.

38. Louise Dupré, op. cit., p. 25.

39. Ibid., p. 14.

40. Louise Dupré à l'émission La poésie n'est pas une solution, France Culture, 2 août 2012.

41. Pierre-Marc de Biasi, Génétique des textes, Paris, CNRS Éditions, coll. «Biblis», 2011, p. 10.

42. Louise Dupré dans Stéphane Lépine, op. cit., p. 13. 


\section{ANNEXE 1 \\ Inventaire descriptif des matériaux d'archives disponibles pour le recueil Les Mots secrets Fonds Louise Dupré (P78), SBAUS}

\section{Chemise 1 «Les Mots secrets»}

1.1. *Tapuscrit partiel de 3 f. (21,6 par $27,9 \mathrm{~cm}^{* *}$; comprend les poèmes 1,2 et 10$)$, sans annotation.

1.2. Tapuscrit partiel de $22 \mathrm{f}$. (poèmes 1-17, 19-22, 24), comportant des annotations à l'encre rouge et à l'encre bleue par Louise Dupré.

1.3. Tapuscrit complet de $29 \mathrm{f}$. (poèmes 1-25, comprenant des doublons des poèmes $13,24,25)$, comportant des annotations à l'encre mauve par Louise Dupré.

Chemise 2 «Les Mots secrets»

2.1. Tapuscrit partiel de $23 \mathrm{f}$. (poèmes 1-23), comportant des annotations à l'encre mauve par Louise Dupré.

2.2. Tapuscrit partiel de $18 \mathrm{f}$. (poèmes $1,2,8,9,12,14-19,21$, $22,24,25$, comprenant des doublons des poèmes $2,22,25$ ), comportant des annotations à l'encre mauve par Louise Dupré.

2.3. Tapuscrit partiel de $10 \mathrm{f}$. (poèmes $1,2,6,11,12,15,19-21,24$ ), comportant des annotations à l'encre mauve par Louise Dupré.

\section{Chemise 3 "Les Mots secrets (annoté par l'éditrice)»}

3.1. Tapuscrit complet de $25 \mathrm{f}$. (poèmes 1-25), comportant des annotations à l'encre noire par Sylvie Massicotte et à l'encre mauve par Louise Dupré.

3.2. Tapuscrit complet de $26 \mathrm{f}$. (poèmes 1-25), sans annotation; il s'agit ici d'un doublon de la copie envoyée à l'éditrice.
On trouve en outre deux feuillets de notes manuscrites à l'encre mauve $(21,2 \times 27,6 \mathrm{~cm})$, sur papier quadrillé $0,5 \mathrm{~cm}$ et un feuillet cartonné de notes manuscrites à l'encre mauve et à l'encre rouge $(10,2 \times 15,2 \mathrm{~cm})$, écrit recto verso.

\section{Chemise 4 «Les mots secrets»}

4.1. Tapuscrit partiel de $16 \mathrm{f}$. (poèmes 1, 2, 4-6, 8, 10, 12-14, 16, 17, 19, 23-25), comportant des annotations à l'encre rouge par Louise Dupré et quelques marques au surligneur jaune.

4.2. Tapuscrit complet de $26 \mathrm{f}$. (poèmes 1-25), comportant des annotations à l'encre rouge et à l'encre mauve par Louise Dupré.

\section{Chemise 5 «Les mots secrets (épreuves)»}

5. Photocopie d'un jeu d'épreuves de $16 \mathrm{f}$. $(21,6 \times 35,6 \mathrm{~cm})$, comportant des annotations à l'encre rouge et à l'encre mauve par Louise Dupré et des annotations allographes en noir.

\section{Chemise 6 «Les mots secrets (mise en scène A. Perrier)»}

Dactylogramme de $35 \mathrm{f}$. du scénario de l'adaptation pour la scène des Mots secrets par André Perrier, daté du 12 juin 2015, comportant des annotations à l'encre noire par Louise Dupré.

On trouve en outre un feuillet de notes manuscrites présentant des suggestions de corrections par Louise Dupré et six feuillets dactylographiés, probablement par André Perrier, sur la réception du spectacle présenté à différentes classes d'élèves du primaire.

\section{ANNEXE 2}

\section{Liste des incipit des poèmes publiés}
1. Au cœur du silence
2. Je connais des mots aussi larges
3. Je joue parfois avec les lettres
4. Certaines phrases font mal
5. Le chagrin ne ménage personne
6. Il y a les larmes des folles tristesses
7. On peut offrir ses mains
8. Le matin se lève toujours trop tôt
9. Les mots du cœur
10. Les poèmes me font rêver
11. Il suffit d'ouvrir ma fenêtre
12. J'entends souvent des paroles
13. Il me faudrait des bras

14. Personne ne me comprend

15. Je ne peux pas expliquer le désordre

16. Souvent il me vient un poème

17. Un enclos dans ma poitrine

18. Ce qui me touche le plus

19. Mais je sais que ma bouche

20. Un jour, j'aurai une maison

21. Il m'arrive d'imaginer des paroles

22. J'ai l'habitude d'écrire

23. Je ne crois plus aux fées

24. Mes plus beaux rêves

25. Je ne trouve pas toujours

(*) Les tapuscrits ne sont pas numérotés. La numérotation que j'applique ici correspond à l'ordre chronologique de la genèse tel que je l'ai reconstitué.

(**) Tous les feuillets des tapuscrits sont de même dimension. S'il y a des variations, les dimensions sont indiquées entre parenthèses. 
Récipiendaire du prix scientifique Anne-Hébert 2018 pour sa thèse de doctorat (Université de Sherbrooke, 2015), ANNIE TANGUAY termine un stage postdoctoral à l'Université du Québec à Montréal sur les pratiques d'écriture d'Anne Hébert et de Louise Dupré, à partir de leurs archives littéraires. Elle a notamment préparé l'édition critique du recueil de nouvelles Le Torrent et des pièces de théâtre de la période 1945 à 1967, qui a paru dans le cinquième tome des Euvres complètes d'Anne Hébert (PUM, 2015).

tanguay.annie.2@courrier.uqam.ca

\section{Transformations poétiques dans Les Mots secrets de Louise Dupré}

Cet article examine les variantes d'écriture de quatre des vingtcinq poèmes du recueil pour adolescents Les Mots secrets de l'écrivaine québécoise Louise Dupré. Cette dernière a publié une vingtaine de livres, dans différents genres littéraires. Les Mots secrets est son seul écrit pour la jeunesse. Plusieurs variantes témoignent du désir de la poétesse d'ajuster sa voix à celle de son public, afin de créer un sentiment de complicité entre le sujetnarrateur et le lecteur. Elle change notamment l'allocution, de la deuxième à la première personne, et recentre son propos sur l'intériorité du personnage. Comme pour ses autres livres, la poétesse écrit en lisant d'autres écrivains, ce qui lui permettra de relancer l'écriture de l'un de ses poèmes.

This article examines the writing variants of four of the twenty-five poems of Quebec writer Louise Dupré's Les Mots secrets, a poetry book for teenagers. Louise Dupré has published about twenty books, in various literary genres. Les Mots secrets is her only book for youth. Several variations testify to the poet's desire to adjust her voice to that of her audience, in order to create a feeling of complicity between the narrator and the reader. She changes the form of speech, from the second person to the first person, and refocuses on the interiority of the character. As is the case for her other books, the poet writes while reading other writers, and this allows her to revive the writing of one of her poems.

Dieser Artikel untersucht die Schreibvarianten von vier der fünfundzwanzig Gedichte Les Mots secrets der aus Québec stammenden Autorin Louise Dupré. Sie hat ungefähr zwanzig Bücher in verschiedenen literarischen Genres veröffentlicht. Les Mots secrets ist ihr einziges Stück Jugendliteratur. Mehrere Varianten zeugen von dem Wunsch der Autorin, ihre Sprache an die des Publikums anzupassen, um ein Gefühl der Verbundenheit zwischen der Erzählerin und ihrer Leserschaft zu schaffen. Sie variiert insbesondere die Anrede von der zweiten zur ersten Person und konzentriert ihren Fokus dann wieder auf die Innerlichkeit der jeweiligen Figur. Was ihre anderen Bücher anbelangt, so schreibt die Autorin, indem sie sich an anderen Schriftstellern inspiriert, wodurch es ihr gelingt, die Arbeit an ihren eigenen Gedichten neu zu beleben.

Este artículo analiza las variantes de escritura de ochenta y cinco poemas de la obra para adolescentes Les Mots secrets de la escritora quebequense Louise Dupré, único texto destinado a los jóvenes, de los veinte libros que esta autora ya lleva publicados. Numerosas variantes dan cuenta del deseo de la poetisa de acordar su voz a la de su público a fin de crear una sensación de complicidad entre el sujeto-narrador y el lector, en primer lugar, pasando su discurso de la segunda a la primera persona y reorientando su enfoque hacia la interioridad de los personajes. Como ha sucedido con otros de sus libros, la poetisa escribe leyendo a otros escritores, lo que le permitirá retomar la redacción de uno de sus poemas.

São examinadas as variantes de quatro dos vinte e cinco poemas da colectânea para adolescentes Les Mots secrets, da escritora canadiana Louise Dupré, do Québec, autora de uma vintena de livros de diversos géneros. Les Mots secrets é a sua única obra destinada à juventude. Certas variantes reflectem o desejo da poetisa de ajustar a voz ao seu público, para criar um sentimento de cumplicidade entre o sujeito-narrador e o leitor. Para isso, ela muda a alocução da segunda para a primeira pessoa e focaliza as suas observações sobre a interioridade do personagem. Como em outros livros, a poetisa escreve sob a influência da leitura de outros escritores, o que se torna palpável na escrita dos seus poemas.

L'articolo esamina le varianti di redazione di quattro dei venticinque poemi della raccolta per adolescenti Les Mots secrets, unico testo per l'infanzia della scrittrice quebecchese Louise Dupré. Diverse varianti testimoniano il desiderio della poetessa di adattare la sua voce a quella del suo pubblico, per creare un sentimento di complicità tra il soggetto narrante e il lettore. Il discorso viene modificato, passando dalla seconda alla prima persona, e ricalibrato sull'interiorità del personaggio. Come per altre sue opere, la poetessa scrive leggendo altri scrittori, e ciò le permetterà di riprendere uno dei suoi poemi che aveva interrotto. 